\title{
Una perspectiva política sobre la evaluación de gerentes en el Estado
}

\author{
lacoviello, Mercedes*
}

\section{Resumen}

Este trabajo intenta explicar desde una perspectiva política las actividades de influencia que caracterizan el proceso de evaluación de gerentes en el Estado. El supuesto del cual se parte es que dados los intereses en juego, tanto el evaluador como el evaluado intentarán afectar los resultados del proceso de evaluación. Si bien esta situación se da en todas las organizaciones, en el contexto del Estado se potencia aún más, especialmente en altos niveles en los cuales interactúan los cuadros políticos y técnicos. El trabajo se centra en el análisis de las condiciones que favorecen el desarrollo de actividades de influencia en los procesos de evaluación de desempeño. A partir de la identificación de estas condiciones, se exploran algunas estrategias posibles para disminuir el nivel de actividades de influencia en el contexto de la evaluación de gerentes en el Estado.

Palabras clave: Evaluación, gerentes, desempeño, gerencia pública.

\section{Recibido: 98-09-30. Aceptado: 99-04-23}

* Licenciada en Economia, Universidad de Buenos Aires. Master in Human Resource Management, University of Illinois, USA. Administradora Gubernamental, Secretaria de la Función Pública, Jefatura de Gabinete de Ministros (Argentina). Miembro del Centro de Estudios de Politica, Administración y Sociedad (CEPAS) y de la Asociación de Administradores Gubernamentales. E-mail: aag@wamani.apc.org 


\section{A Political Perspective on the Evaluation of Managers in the State}

\section{Abstract}

This work attempts to explain, from a political point of view, the activities of influence that characterize the process of evaluating managers in the State. The initial supposition is that, given the interests at play, the evaluator as well as the person who is evaluated will try to affect the results of the evaluation process. Though this situation occurs in all organizations, in the context of the State it becomes even stronger, especially at high levels where political and technical scenes interact. This work focuses on the analysis of conditions that favor the development of activities of influence in the processes of performance evaluation. After identifying these conditions, the article explores some possible strategies for reducing the level of activities of influence within the context of evaluation of managers in the State.

Key words: Evaluation, managers, performance, public management.

\section{Introducción}

Dentro del campo de la Administración de recursos humanos, la evaluación de desempeño es uno de los temas más investigados, ya que el producto de este proceso se utiliza para tomar decisiones centrales en la organización. La evaluación de desempeño provee feedback a los empleados, indicándoles los aspectos a reforzar para que su desempeño se oriente a los objetivos de la organización. Medidas precisas del desempeño de los empleados resultan indispensables para validar los procesos de selección, para tomar decisiones respecto de las promociones y para establecer los niveles de remuneración.

Dada la importancia de contar con una medida precisa del desempeño, la mayoría del trabajo de investigación en el área se ha focalizado en problemas de medición, desarrollo de escalas, formato de escalas, o en la reducción de los errores en los tests o en las apreciaciones de los evaluadores. Más recientemente, la actividad de investigación se ha orientado a los procesos cognitivos involucrados en las evaluaciones. Las etapas de adquisición, codificación, almacenamiento y recuperación de información se analizan a fin de comprender las capacidades de los supervisores como evaluadores. Algunos estudios también se han centrado en los aspectos emocionales del proceso de evaluación de desempeño que desdibujan la objetividad buscada. Todas estas diferentes líneas de investigación tienen un tema en común: tratan de identificar los factores que afectan la precisión 
en la medición del desempeño. La premisa detrás de este enfoque, que a modo de simplificación denominaremos perspectiva racional, es que existen niveles reales de desempeño de los evaluados, y que estos niveles pueden ser evaluados objetivamente, si bien con cierto nivel de error.

En los últimos años este enfoque ha sido cambiado a partir de los estudios realizados desde una perspectiva socialpsicológica. La evaluación de desempeño se ve como un proceso social y de comunicación, más que como una herramienta de medición. El supuesto es que el comportamiento del evaluador y del evaluado está dirigido a ciertos objetivos, y que las evaluaciones de desempeño implican una interacción social. El contexto pasa a ser absolutamente relevante bajo este supuesto, ya que provee el entorno en el cual se desarrolla este proceso social.

La perspectiva política que se propone en este trabajo aplica conceptos de la literatura sobre influencia social y política organizacional para explicar el comportamiento de evaluador y evaluado en el proceso de evaluación de desempeño. El supuesto es que las organizaciones son entidades políticas, y que las partes clave involucradas en todo proceso de decisión actúan en su propio interés.

Cuando nos referimos a cuestiones políticas en este contexto, no estamos hablando de política partidaria sino de política organizacional. En este sentido, la actividad política se refiere a cualquier intento por parte de los individuos de proteger sus propios intereses ante cursos de acción alternativos (Pfeffer, 1992). En las organizaciones públicas, la política parti- daria se entremezcla con la política organizacional en el sentido técnico definido, ya que los niveles superiores del gobierno están cubiertos por funcionarios políticos que necesariamente introducirán sus intereses partidarios en el juego político interno.

La perspectiva política se aparta de los supuestos básicos de las líneas de investigación tradicionales respecto de la evaluación de desempeño. Cardy \& Dobbins (1994) presentan una interesante diferenciación entre las perspectivas racional y política en términos de los objetivos de la evaluación, el rol de los participantes, la definición del desempeño, la información a evaluar, el proceso de decisión (Cuadro 1).

La perspectiva racional supone que la obtención de medidas precisas es el objetivo del sistema y del evaluador. En consecuencia, la precisión ha sido tradicionalmente la variable para evaluar la efectividad del sistema de evaluación de desempeño. Evaluadores y evaluados se consideran como entidades pasivas en el proceso de evaluación. Se entiende al evaluador como un juez sin intenciones predeterminadas, si bien con cierta falibilidad en cuanto a su capacidad de almacenar, recordar y evaluar el desempeño de los evaluados. Se supone bajo este esquema que si bien los evaluadores son falibles y tendenciosos, un mejor instrumento de evaluación puede mejorar significativamente la precisión de las evaluaciones. El desempeño del evaluado es claramente definido a partir de los estándares que surgen de la definición de su puesto. La información a evaluar es concreta y precisa, referida estrictamente a los comportamientos relacionados con el 
Cuadro 1

Perspectiva Racional y Perspectiva Política

\begin{tabular}{lcl} 
& Perspectiva racional & Perspectiva política \\
\hline Objetivo & precisión & utilidad \\
Roles de los participantes & pasivos & motivados \\
Elemento central & medición & gerenciamiento \\
Desempeño del evaluado & definido & impreciso \\
Información a evaluar & molecular & global \\
Proceso de decisión & inductivo & deductivo \\
\hline
\end{tabular}

Fuente: Cardy \& Dobbins, 1994.

puesto, y no relacionada con impresiones generales respecto del evaluado. Se trata de un proceso inductivo en el cual la evaluación agregada es producto de la suma y promedio de los comportamientos específicos evaluados.

Por otro lado, la perspectiva política entiende el proceso de evaluación de desempeño como una herramienta gerencial para obtener resultados que son deseables de acuerdo al contexto político y a la agenda del evaluador. Si los resultados del proceso de evaluación se alejan del ideal de precisión y objetividad del modelo racional, es porque tanto evaluador como evaluado persiguen otros objetivos. El objetivo de la evaluación de desempeño en este contexto se mide en términos de utilidad, de maximización de beneficios sobre costos. Tanto evaluador como evaluado están motivados para influenciar el proceso de evaluación y los resultados del mismo. El tema no es ya la medición, sino la utilización del proceso como herramienta para controlar el comportamiento del evaluado. El desempeño no se entiende como una construcción precisa, pues lo que se evalúa como positivo o negativo dependerá de los intere- ses del evaluador y del contexto político en la organización. La evaluación no contemplará entonces información especifica acerca del desempeño, sino una impresión general respecto del evaluado que definirá la evaluación de cada una de las dimensiones establecidas en el sistema.

El proceso de evaluación se interpreta como un proceso político en el cual los actores intentarán utilizar los medios a su alcance para afectar el proceso de decisión de manera de favorecer sus propios intereses. Veamos entonces cuales son los intereses de ambas partes en la evaluación de desempeño, y cuáles son los medios con los que cuentan para afectar el proceso y sus resultados.

El interés del evaluado es obtener una calificación lo más alta posible, ya que hay decisiones importantes que dependen del nivel de calificación obtenido: retribución, posibilidades de ascenso, etc. Para lograr sus objetivos, el evaluado tiene incentivos para ejercer actividades de influencia sobre el evaluador. Una estrategia posible es manejar las impresiones que el evaluador tiene, llevándolo a percibir una imagen mejor que la real du- 
rante el proceso de evaluación. En este contexto el concepto de manejo de impresiones (impression management) se refiere a cualquier comportamiento que altera o mantiene la imagen de la persona a los ojos de otra, y que se dirige a la obtención de algún objetivo considerado valioso (Villanova y Bernardin, 1989). Otra alternativa es intentar modificar el resultado de la evaluación, utilizando toda su influencia para hacer que el evaluador se sienta obligado a modificar un nivel de calificación que pudiera resultar perjudicial.

Dentro del manejo de impresiones se puede distinguir entre tácticas orientadas al puesto, en las cuales el evaluado intenta promoverse a fin de parecer mas competente en el trabajo, y tácticas orientadas al supervisor, que se dirigen directamente a congraciarse con el evaluador tratando de agradarle (Ferris et al., 1994). Ejemplos de tácticas orientadas al puesto podrian ser trabajar hasta tarde en la oficina para que el supervisor lo vea como una persona esforzada, o hacer que el supervisor piense que uno es el único responsable por hechos positivos que en realidad son responsabilidad de un grupo de trabajo. Entre las tácticas orientadas al supervisor se incluirian, por ejemplo, interesarse en la vida personal del superior, 0 alabarlo y resaltar sus logros. Un listado más exhaustivo sobre esta tipología de tácticas orientadas al puesto y al supervisor se puede ver en el Cuadro 2.

De acuerdo a la investigación empírica en el área, en el contexto de la evaluación de desempeño las tácticas orientadas al supervisor resultaron más apropiadas y efectivas para lograr mejores calificaciones ${ }^{1}$. La razón de esta diferencia parece estar en el efecto del factor afectivo en el proceso de evaluación. El afecto del supervisor hacia el evaluado genera un incremento en el efecto halo, que se refleja en mayores calificaciones para igual nivel de desempeño. En las tácticas orientadas al supervisor el objetivo es resultar más agradable, y esto tiene un efecto positivo en el afecto del supervisor hacia el subordinado. En las tácticas orientadas al puesto el objetivo es parecer mas competente, y estos comportamientos tienden a disminuir el afecto del superior por el subordinado.

El evaluador también tiene intereses asociados a los resultados de la evaluación. Su propia imagen en la organización depende del nivel de desempeño de sus subordinados, por lo cual le conviene también que los resultados de la evaluación sean positivos. Por otro lado, evaluaciones indulgentes bajan el nivel de conflicto con los subordinados. La evaluación de desempeño es una herramienta de gerenciamiento que los supenvisores utilizan para mejorar el desempeño futuro de

1 Es interesante, porque en el contexto de las entrevistas de selección, el resultado de la investigación empírica es el contrario (Ferris et al., 1994). Los seleccionadores realizaron evaluaciones mas favorables de los candidatos que se auto-promovian basados en su experiencia laboral (táctica orientada al puesto) que los candidatos que intentaban congraciarse con ellos (táctica orientada al supervisor). 


\section{Cuadro 2}

\section{Tácticas para el Manejo de Impresiones}

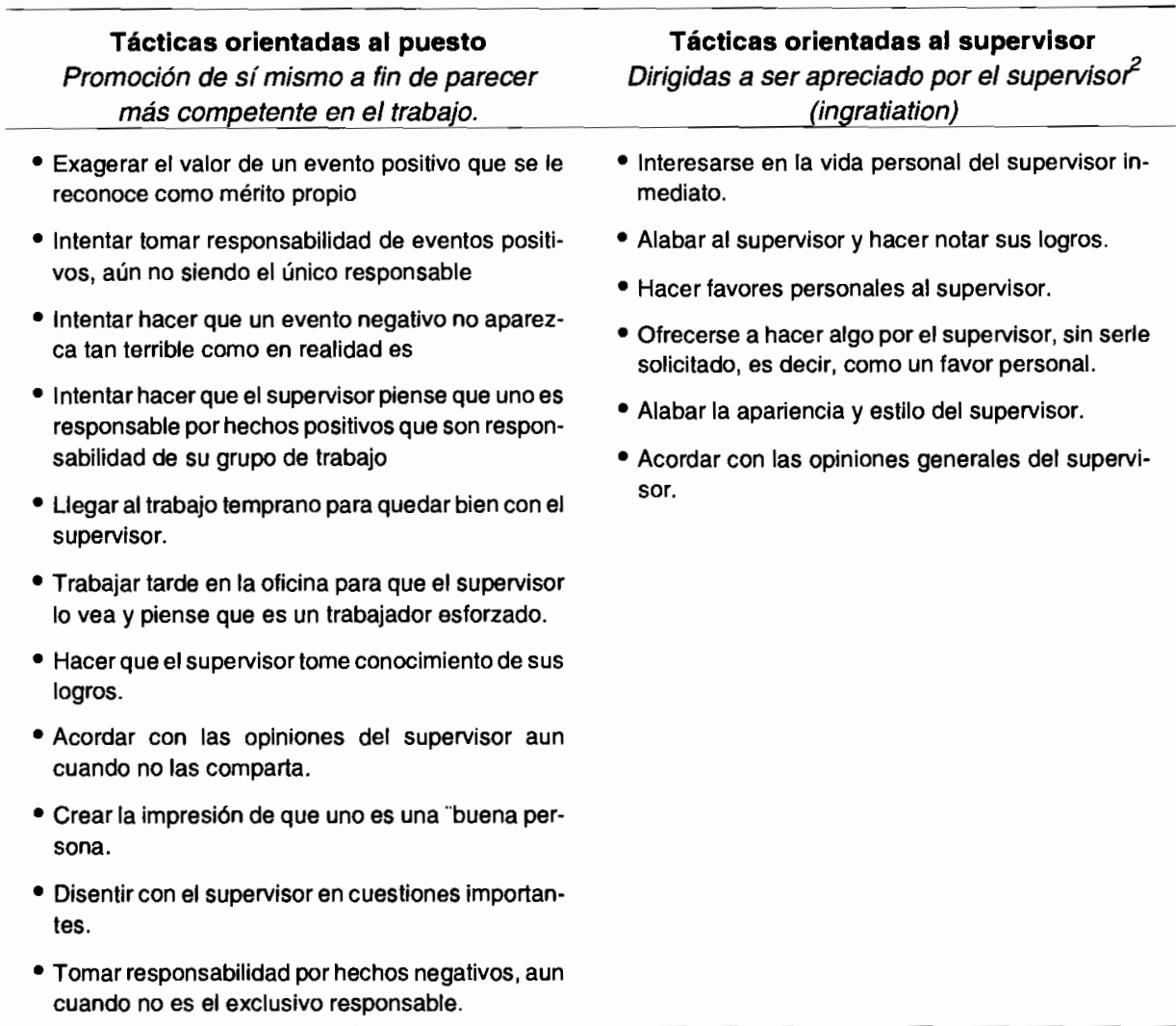

Fuente: Ferris et al., 1994.

sus subordinados. Esta estrategia motivadora no necesariamente coincide con la evaluación objetiva del desempeño del empleado. Longenecker, Gioia y Sims (1987), a partir de entrevistas a 60 ejecutivos, concluyeron que éstos manipulaban las evaluaciones con diferentes obje- tivos y que se consideraban con derecho a hacerlo como parte de sus atribuciones gerenciales. Los supervisores pueden inflar las evaluaciones para reconocer el esfuerzo y motivar al empleado aun cuando los estándares no se hayan alcanzado, o para proteger a sus subordinados supervisor. 
ante situaciones de downsizing ${ }^{3}$. Los supervisores también pueden bajar el nivel de calificación para dar una señal de alerta a un subordinado para que cambie su conducta, o para generar los antecedentes sobre los cuales basar una futura degradación o despido.

Estas estrategias para manipular las evaluaciones son aplicables a todas las organizaciones, pero en el contexto de la evaluación de funcionarios técnicos en el Estado por parte de sus superiores políticos toma ciertas características particulares que analizaremos a continuación.

\section{La evaluación de gerentes en el Estado como campo de actividad política}

En las organizaciones públicas el diseño e implementación de las estrategias de recursos humanos se desarrolla en un medio altamente politizado, y está determinado por la contradicción de intereses entre diferentes grupos de poder. Si bien en todas las organizaciones los procesos decisorios están sujetos a procesos de influencia, en las organizaciones públicas esa situación se exacerba por las características particulares que presentan y por la interacción entre los cuadros políticos y técnicos.
El análisis realizado sobre la perspectiva política aplicada a los procesos de evaluación de desempeño resulta particularmente útil para explicar el juego político que se da en la evaluación de niveles jerárquicos en los organismos públicos (lacoviello, 1996). Veamos cuáles son los intereses en juego en el contexto de la evaluación de funcionarios de línea por parte de sus superiores políticos.

El interés de los funcionarios políticos en relación con las políticas de recursos humanos consiste en preservar su capacidad de decisión para mantener bajo su cargo personas de confianza, de manera de asegurar que sus directivas sean implementadas por el aparato burocrático. Dado que son responsables frente al público por los resultados de su mandato, tienen interés en preservar las posiciones críticas para personas políticamente comprometidas. Las evaluaciones de desempeño estarán signadas por este objetivo primordial, de modo que habrá una tendencia a favorecer en las evaluaciones a los funcionarios con afinidad política (no necesariamente partidaria), o perjudicar a los funcionarios que no la tengan para modificar su comportamiento.

La posibilidad de manejar el proceso de evaluación y sus resultados dependerá del grado de autonomía con que

3 En el informe de la General Accounting Office (GAO, 1993) acerca del sistema de evaluación de desempeño en el Federal Civil Service se encontró que en el marco de reforma en curso ( $\mathrm{Na}$ tional Performance Review), los supervisores tienden a aumentar las calificaciones para proteger a sus subordinados en vez de proveerles un feedback preciso que les permita mejorar su performance. 
puedan tomar este tipo de decisiones. $\mathrm{Si}$ bien los funcionarios políticos representan los niveles superiores de la organización, el sistema de mérito plantea regulaciones y procedimientos que limitan su margen de acción. Además, hay un cierto grado de responsabilidad hacia las máximas autoridades, tanto respecto de las formalidades de la evaluación corno del efectivo gerenciamiento del desempeño de sus subordinados. Otro factor importante que permite a los políticos influir el proceso de evaluación es el hecho de tener el control sobre los recursos dentro de suárea, ya que ésta es una herramienta que puede afectar los resultados de las evaluaciones. La asignación de recursos define en parte la consecución de objetivos por parte de los subordinados, e indirectamente afecta sus calificaciones.

Por su parte los funcionarios de línea tienen como interés fortalecer su posición en la organización a través de altas calificaciones y evitar posibles discrecionalidades por parte de sus superiores políticos en las evaluaciones. La formalización de los procesos de evaluación provee a los funcionarios de línea una efectiva defensa contra posibles manejos por parte del superior (Daley, 1992). Sus conocimientos sobre los procedimientos y sobre el funcionamiento de la organización también representan una herramienta importante para manejar las impresiones del superior. Además, su situación de intermediación en el flujo de información desde el resto de la organización hacia el funcionario político les permite cierto manejo de la información de acuerdo a sus intereses.
Con respecto al manejo de impresiones, se podría esperar que las tácticas orientadas al puesto resulten más accesibles para el funcionario evaluado, ya que cuenta con la ventaja de una menor familiaridad del funcionario político respecto del funcionamiento de la organización. Es factible en este contexto manejar la información de manera de aparecer como responsable de hechos positivos, o estar presente en los momentos críticos para crear una imagen favorable en el funcionario político.

Las tácticas orientadas al supervisor parecieran menos accesibles en un contexto de mayor distancia y menor tiempo de interacción como es el que generaimente se da en la relación entre el nivel político y el nivel técnico. En este sentido, los funcionarios técnicos se encontrarian en desventaja para implementar sus tácticas de influencia, ya que se encuentran limitados en el uso de las tácticas más efectivas en el contexto de la evaluación de desempeño. Por supuesto que esta situación excluye a los colaboradores inmediatos del político, pero en este caso la afinidad pre-establecida hace innecesario el comportamiento "oportunista" del evaluado, que ya cuenta con una imagen favorable

Es importante también la red de contactos que generan los funcionarios de línea a través de su permanecia en la organización, lo cual les permite tener aliados a los que recurrir ante posibles resultados negativos de las evaluaciones. Dentro de la organización, un sector que puede brindarles apoyo ante situaciones conflictivas es el área de Recursos Humanos. Si bien se trata en general de áreas profesionales con un rol neutral en 
los conflictos que pudieran generarse, pueden jugar a favor del funcionario de línea haciendo cumplir los procedimientos ante intentos de manipulación por parte del político. La situación de árbitro ante estas situaciones no es fácil, ya que el área puede recibir presiones políticas respecto de las decisiones a tomar.

Si bien los miembros de la coalición externa tienen menos acceso al proceso de evaluación de desempeño por ser un proceso interno, pueden pasar a tomar parte activa en el proceso cuando son incorporados por el evaluado como parte de una alianza, o para implementar una estrategia de presión contra el evaluador. El funcionario de línea que se siente amenazado por una posible evaluación negativa podría apelar a los grupos de interés relacionados con la organización. Estos grupos apoyarán al funcionario evaluado si éste representa un factor de influencia para favorecer sus intereses en la organización. Esto genera en algunos casos un proceso de lobby en el cual grupos organizados actúan como "sponsor" de candidatos para la designación o promoción en niveles de decisión, de manera que sus intereses estén representados (Shafritz et al, 1992). También podría recurrir al partido político al que pertenece, ya que cada partido respaldará las decisiones que favorezcan la posición de los funcionarios vinculados a su organización. Las organizaciones sindicales también podrían proveer apoyo, ya que están interesadas en proteger los puestos y prerrogativas de sus miembros y en general intentan limitar la discreción gerencial en materia de promociones, compensación y separación del puesto. Sin embargo, en los altos niveles de la organización hay menor grado de sindicalización, lo que genera menor incidencia de este grupo en las evaluaciones de funcionarios de línea en los niveles jerárquicos.

La evaluación de gerentes en el Estado se da entonces en un contexto necesariamente politizado debido a las características de la organización y a las características del proceso de evaluación de desempeño en sí, agravado por el alto nivel en que se desarrolla este proceso. Políticos y funcionarios de línea tienen objetivos que pueden ser contradictorios respecto de los resultados de la evaluación de desempeño, y tienen acceso a medios para afectar el proceso de evaluación debido a la posición que ocupan dentro de la organización y sus relaciones con actores externos. En el Cuadro 3 se resumen los intereses de cada parte, y los medios con los que cuentan para influir el proceso de evaluación.

La perspectiva política planteada provee una descripción realista del proceso de evaluación de gerentes en el Estado. Al mismo tiempo, el modelo racional y su objetivo de precisión en la medición del desempeño provee el ideal teórico del proceso de evaluación (Cardy \& Dobbins, 1994). Las dos perspectivas son complementarias, y ambas son útiles para determinar posibles mejoras en la evaluación de desempeño. El objetivo es determinar como maximizar la precisión en las evaluaciones, dada la realidad de los comportamientos políticos que las afectan.

El resto del trabajo intenta ir más allá del aspecto descriptivo del modelo político: el objetivo es determinar estrategias que permitan reducir el nivel de politización del proceso de evaluación a partir de la identificación de las variables que 


\section{Cuadro 3}

Intereses y Medios de Influencia

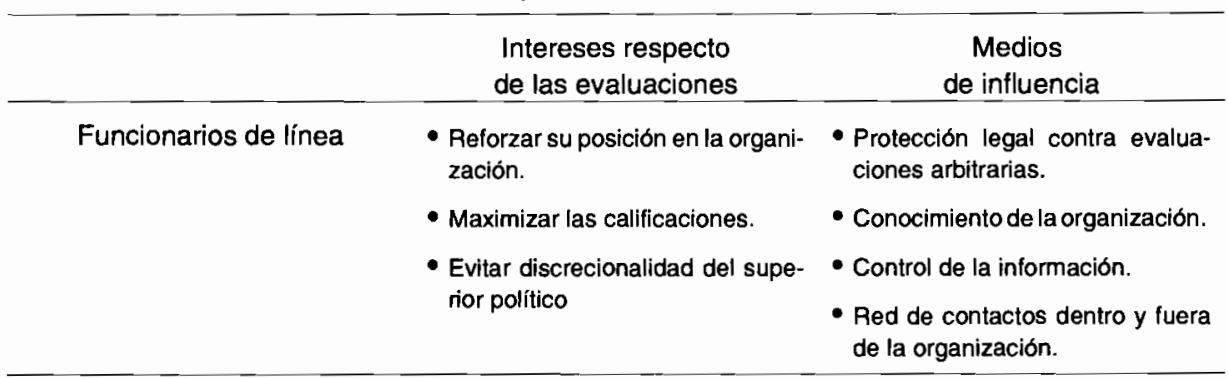

favorecen el desarrollo de comportamientos de influencia.

\section{Factores que favorecen el desarrollo de comportamientos de influencia}

La literatura sobre política organizacional identifica varios factores del contexto los cuales hacen que los procesos organizacionales sean más susceptibles a comportamientos de influencia. Estos factores están moderados por las diferencias individuales, ya que no toda persona tendrá la misma tendencia a comportamientos políticos, aun bajo las mismas condiciones ambientales (Ferris \& Judge, 1991). Al mismo tiempo, este tipo de comportamiento requiere cierto grado de aptitud política que no todos los individuos tienen.

Sin ignorar estas diferencias individuales, el resto del trabajo se centrará en los factores organizacionales bajo los cuales es más factible que se desarrollen comportamientos de influencia. En las secciones siguientes se describirán estos factores, aplicándolos al contexto de la evaluación de funcionarios técnicos por parte de sus superiores políticos.
Los factores que cuentan con cierto grado de consenso en la literatura como antecedentes de política organizacional son los siguientes:

- importancia del asunto en cuestión,

- interdependencia y contradicción de intereses entre las partes,

- grado de ambigüedad del proceso, incertidumbre respecto de los resultados,

- grado de formalización del proceso

- instrumentalidad de los comportamientos de influencia

- grado de centralización del proceso de decisión

- nivel jerárquico en la organización

Como se verá, algunos de estos factores son propios de la naturaleza del proceso de evaluación de desempeño, mientras otros dependen de la situación específica en la cual este proceso tiene lugar. El nivel de politización podría ser manipulado trabajando sobre estas variables contextuales.

\subsection{El poder se aplica}

\section{a las cuestiones importantes}

El poder es un recurso valioso, y por lo tanto no se aplica a cuestiones triviales (Pfeffer, 1992). El nivel de impor- 
tancia de un asunto se define en términos de las partes interesadas. Enormes dosis de poder se pueden invertir en cuestiones objetivamente irrelevantes como la ubicación de una oficina. Lo que cuenta es la importancia que las partes afectadas asignan al asunto.

Si la decisión tiene consecuencias relevantes, relacionadas con la distribución de recursos escasos en la organización, habrá mas incentivos para que los participantes intenten afectar el proceso de toma de decisiones (Salancik \& Pfeffer, 1976). Entonces, el proceso de evaluación de desempeño en sí mismo es susceptible de influencias políticas dada la importancia de las decisiones para las partes. Tanto el evaluador como el evaluado -y también la organización- tienen intereses vitales en juego. Desde el punto de vista del evaluado, hay decisiones importantes que dependen del resultado de la evaluación. El uso que se da a las calificaciones está directamente relacionado con la trascendencia asignada al proceso de evaluación, y habrá mayor nivel de actividad política cuando los resultados de la evaluación estén asociados a consecuencias en el status económico, social y político del evaluado en la organización. Este es el caso cuando las promociones, las remuneraciones y los despidos están relacionados con los resultados de la evaluación, situación típica de los sistemas de mérito propios de la función pública.

La importancia percibida por las partes respecto del proceso será mayor si las evaluaciones se realizan con poca frecuencia. Como se mencionara antes, hay intereses cruciales para las partes que dependen de la evaluación de personal.
Si todos estos intereses dependen de un único evento que no se repetirá en el corto plazo, habrá una mayor percepción de la irreversibilidad de la decisión a tomar. En los sistemas de mérito en las organizaciones públicas, las decisiones de promoción y remuneración se toman en función de la evaluación anual, que se transforma en un evento de gran importancia en la organización.

\subsection{Actores interdependientes con objetivos contrapuestos}

Cuando hay interdependencia, la posibilidad de obtener los resultados deseados en una determinada situación requiere de la capacidad de influir a la otra parte (Pfeffer, 1992). En el contexto de la evaluación de desempeño, evaluador $y$ evaluado dependen uno de otro. El bienestar de los evaluados depende de las calificaciones determinadas por el supervisor. Al mismo tiempo, el supervisor depende de la información que proveen los evaluados para producir la evaluación. Esta interdependencia hace que cada una de las partes esté en situación de ejercer influencia sobre la otra.

Además del nivel de interdependencia, lo que define el uso del poder en las organizaciones es que existan diferencias de criterio acerca de la decisión a tomar. Habrá mayor nivel de actividad política cuando se combinan interdependencia y disenso, ya que las partes dependen una de otra para obtener los resultados buscados y tienen preferencias divergentes acerca de estos resultados (Welsh \& Slusher, 1986). En el contexto de la evaluación de desempeño las dos condiciones se cumplen. Para la organización es importante obtener información 
precisa sobre el desempeño de sus integrantes a fin de lograr una óptima distribución de recursos. Para el evaluado, el problema es maximizar su bienestar, que está estrechamente relacionado al nivel de calificación obtenida. Para el evaluador, el tema es utilizar el proceso de evaluación como una herramienta para dirigir la conducta de sus subordinados de acuerdo a sus objetivos.

Si nos centramos en la evaluación de gerentes de línea por parte de sus superiores políticos, habrá también otros intereses en juego. Cuando no hay afinidad entre evaluador y evaluado, la politización del proceso se potencia aún más. Para el funcionario político, la instancia de evaluación representa una posibilidad de explicitar el disenso con el subordinado, debilitando su posición en la organización. Para el funcionario de línea, la evaluación representa una situación de peligro ante la cual desarrollará todas las estrategias posibles para evitar consecuencias negativas. Los contactos con otras autoridades de la organización -o con actores externos-que de alguna manera influyan sobre la decisión del superior directo, son un recurso importante en esta situación.

Además de la divergencia entre los objetivos del evaluador y del evaluado, los objetivos de la organización respecto de la evaluación de desempeño no siempre son consistentes entre sí (Birkenhoff \& Kanter, 1980). Por un lado, las evaluaciones deberian ser una fuente de información precisa acerca del desempeño de los miembros de la organización, a partir de la cual se toman decisiones centrales respecto de los recursos humanos. Las evaluaciones se utilizan con fines administrativos, para establecer criterios de selección, decidir promociones, pago de incentivos, y despidos. Por otro lado, deberían proveer feedback a los empleados para mejorar su desempeño, proveyendo una herramienta valiosa para mejorar la productividad y motivar a los empleados. No siempre es posible servir a todos estos objetivos al mismo tiempo, lo cual deja abierta la posibilidad a los participantes de decidir cuales serán los objetivos privilegiados en el proceso de evaluación.

\subsection{Situaciones inciertas y ambiguas}

Según Salancik, Pfeffer \& Leblebici (1976), cuando hay estándares consensuados y bien definidos, los resultados del proceso de decisión se basan en estos estándares. En ausencia de estándares compartidos, las decisiones responderán a procesos de influencia social. Si hay una clara operacionalización de objetivos, habrá poco margen de maniobra para el decisor, y menos incentivos para que la parte afectada intente estrategias políticas para afectar el proceso. Para que los objetivos sean operativos, tienen que ser consensuados, estables y traducibles en términos cuantitativos.

Esta idea acerca de los procesos de decisión en general se puede aplicar al contexto de la evaluación de desempeño. Estándares específicos y bien definidos para evaluar el desempeño reducirán el nivel de actividades de influencia en el proceso de evaluación. Mayor especificidad aumenta la verificabilidad, inhibiendo las distorsiones por parte de evaluador $y$ evaluado. Cuando hay medidas tangibles del desempeño, los individuos saben lo que se espera de ellos, y pueden demos- 
trar sus logros. Del lado del evaluador, criterios más específicos de evaluación afectarán la percepción del supervisor acerca de la importancia que se otorga a la precisión en las evaluaciones. Los criterios ambiguos abren la posibilidad al manejo de impresiones por parte del evaluado, y a la manipulación de las calificaciones por parte del evaluador (Villanova \& Bernardin, 1989). En los niveles superiores en las organizaciones públicas es difícil operacionalizar los objetivos de cada área y determinar los estándares para evaluar a cada funcionario, lo que crea un ambiente propicio para actividades de influencia.

El proceso de evaluación estará más sujeto a este tipo de comportamientos si hay factores fuera del control de los evaluados que pueden afectar el nexo entre el nivel de desempeño y el resultado de la evaluación. Si hay variables externas que afectan el desempeño, o si la evaluación depende de juicios subjetivos del evaluador, los resultados del proceso resultarán ambiguos e inciertos para los evaluados, favoreciendo el manejo de impresiones (Wood \& Mitchell, 1981). Ambas variables se refuerzan entre sí. Si el nivel de desempeño depende de variables externas, el evaluador se encontrará en la situación de realizar juicios subjetivos para discernir hasta que punto el evaluado fue responsable por un bajo desempeño del área, o si la falla se debió a factores externos. Esta situación hace posible que el evaluado intente manejar las impresiones de su supervisor, intentando asignar el problema a factores ajenos a su control para evitar que se lo haga reponsable del problema.

\subsection{Formalización de los procesos de decisión}

Este factor esta relacionado con el anterior: se puede decir que si la formalización del proceso de evaluación disminuye el grado de ambigüedad, entonces debería disminuir también el nivel de actividad política. Milgrom y Roberts (1992) sostienen que si se establecen reglas y procedimientos fijos que deben ser respetados, habrá menos actividad de influencia en los procesos de decisión. Los procedimientos formales siven como un medio de control del comportamiento, $y$ hacen que los individuos vean los comportamientos políticos como menos efectivos (Ferris \& Judge, 1991). Mintzberg (1983) identifica la informalidad con un antecedente fundamental del comportamiento político. El sistema informal de influencia política se pone en juego cuando los sistemas de autoridad, ideología y expertez fallan. La actividad política surge cuando hay "agujeros" en estos sistemas formales, y cuando el individuo anticipa que sus intereses no serán satisfechos a través de los mismos.

En el contexto de la evaluación de desempeño es posible asumir que un mayor grado de formalización de los procedimientos reducirá la ambigüedad percibida por las partes, dejando menos espacio para comportamientos políticos. Los evaluadores tendrán que atenerse a ciertas normas para justificar sus decisiones en términos de ciertas reglas, y los evaluados tendrán menos espacio para manejar las impresiones de sus supervisores.

Dada la diversidad de organismos públicos, es muy difícil lograr una estandarización eficiente de los sistemas de evaluación de desempeño. En el marco 
de la reforma del Federal Civil Service, por ejemplo, uno de los cambios propuestos para el sistema de recursos humanos en el programa Reinventing Government es precisamente la flexibilización de los sistemas de evaluación de desempeño, permitiendo a cada agencia desarrollar sus propios programas de evaluación que contempien sus características especificas (Gore, A., 1993) ${ }^{4}$. Esta tendencia a una mayor flexibilidad busca aumentar la eficiencia de las evaluaciones de desempeño como herramientas de gerenciamiento, pero al mismo tiempo favorece el desarrollo de actividades de influencia. En el caso de los niveles gerenciales en el Estado, asociados a objetivos más ambiguos y tareas no rutinarias, es aún más complicado lograr una estandarización de la evaluación de gerentes.

\subsection{Instrumentalidad de los comportamientos de influencia}

Los individuos se involucran en actividades de influencia siempre que las mismas sean efectivas para la obtención de sus objetivos. La decisión de desarrollar una estrategia de influencia se basa en la probabilidad subjetiva de que ese comportamiento sea exitoso para la obtención del resultado deseado (Ferris et al., 1989). El sistema de recompensas de la organización afectará directamente esta probabilidad subjetiva de éxito. Si el contexto se percibe como altamente político por parte de los individuos, y la organización les permite obtener lo que desean al comportarse políticamente, la politización del sistema se realimenta. En cambio, si los individuos perciben un contexto hostil a la utilización de estrategias de influencia, las verán como una amenaza a sus carreras, y disminuirán los incentivos a comportarse políticamente.

Las organizaciones pueden modelar los sistemas de valores a través de distintos medios, entre los cuales el sistema de selección juega un rol importante. Un contexto político atraerá a candidatos con mayor probabilidad de presentar este tipo de comportamiento. Otro modo de consolidar el sistema de valores de la organización es el sistema de socialización. Los nuevos miembros de la organización observan el comportamiento de sus superiores y de alguna manera imitan su comportamiento. Si ven a sus supervisores desarrollando actividades de influencia, y que estas actividades resultan exitosas, imitarán este comportamiento entendiendo que es el modo característico de proceder en la organización ${ }^{5}$. Entonces, los comportamientos políticos se refuerzan a partir de los criterios de selección, el proceso de socialización y el sistema de recompensas en la organización.

Estas propuestas del Informe Gore se basan en el estudio realizado por la General Accounting Office en febrero de 1993, cuyo título es precisamente La evaluación de desempeño en el Estado: Las agencias necesitan mayor flexibilidad para diseñar sus sistemas (GAO, 1993)

Ferris et al. (1991) desarrollaron estos conceptos partiendo de la aplicación de la teoría del aprendizaje social de Bandura, y aplicándolos al ámtito de la transmisión de valores en el trabajo. 
En niveles gerenciales del Estado, los funcionarios de línea están en directo contacto con superiores políticos. Dentro de la actividad política es central el uso de actividades de influencia. Es bastante probable que el superior en este caso tome decisiones administrativas internas con una lógica similar a la requerida por su actividad política. En este contexto el funcionario de línea a ser evaluado responderá con comportamiento similar, realimentando el proceso.

\subsection{Centralización de la toma de decisiones}

No hay consenso en la literatura acerca de cómo juega este factor en el desarrollo de actividades políticas. Milgrom y Roberts (1992) proponen una relación positiva entre centralización y actividades de influencia. El argumento es que para que las actividades de influencia se desarrollen, es necesario que el poder de decisión esté concentrado en una unidad o en una persona, y que las partes tengan un canal de comunicación con el decisor mientras la decisión está siendo tomada.

Partiendo de una perspectiva diferente, Pfeffer (1992) dice que la centralización puede asociarse con un cierto nivel de consenso en la organización. La centralización podría reflejar un consenso entre los miembros de la organización, y por lo tanto podría hacer que se sientan más libres de expresar opiniones diver- gentes, con lo cual habria menos incentivos para las actividades de influencia.

Estas dos perspectivas parten de diferentes conceptos de centralización, pero ambas son útiles para explicar los aspectos políticos de la evaluación de desempeño en el Estado. Siguiendo el primer argumento, los evaluadores generalmente tienen el poder de tomar las decisiones acerca de las evaluaciones por sí mismos -o con muy poca participación de otros miembros en la organización- ${ }^{6}$, y los evaluados tienen acceso al evaluador durante el proceso de evaluación. Estas características conducirían entonces a un mayor nivel de comportamiento político. Siguiendo el segundo argumento podríamos decir que si las decisiones de evaluación se concentraran en un nivel más alto en la organización -o si hubiera una efectiva revisión por un nivel superior o por una agencia externa-, se generaría una mayor uniformidad de criterios de evaluación y disminuiría el nivel de politización del proceso.

\subsection{Nivel dentro de la estructura jerárquica de la organización}

Según Pfeffer (1992), a más alto nivel dentro de la organización, mayor frecuencia en la utilización de poder e influencia. Esto se relaciona con la importancia de las decisiones a tomar, que siempre será mayor cuanto más alto el nivel en la organización. Bajo condiciones de alta profesionalización y complejidad evaluador mediante una revisión de las calificaciones por el nivel superior al supervisor, estas revisiones suelen ser puramente formales (Steimberg \& Burke, 1986) 
funcional los criterios de evaluación se tornan más subjetivos, como es el caso en los niveles gerenciales de los organismos públicos. En este contexto, los criterios de evaluación pasan a ser la conformidad con las normas de la organización, $y$ las preferencias del supervisor. Además, aquellos niveles con mayor responsabilidad en la organización serán los que tienen mayores incentivos a desarrollar actividades de influencia, ya que tienen mucho más que perder si no logran sus objetivos respecto del proceso de evaluación (Ferris \& Judge, 1991). Murphy y Cleveland (1995) señalan que es justamente en los niveles jerárquicos de la organización donde hay más probabilidad de politización de los procesos de evaluación, y al mismo tiempo es donde más se necesitaría contar con evaluaciones precisas del desempeño, dada la relevancia de las decisiones que se toman en estos niveles.

Como se puede ver, los factores que se identificaron como antecedentes de actividad política en las secciones anteriores juegan un rol mayor en los niveles superiores de la organización, generando mayor grado de actividad politica. Así, el sistema de evaluación de desempeño es particularmente crítico en términos del desarrollo de actividades de influencia en altos niveles de la organización.

Cualquier sistema de evaluación deja espacio para ciertos juicios subjetivos. El problema surge en los niveles jerárquicos altos de los organismos públicos, que deben ser evaluados por niveles políticos. Esta situación es incómoda para ambas partes: deja a los funcionarios de línea en una situación de alta de- pendencia de sus superiores políticos, y éstos a su vez se ven en la difícil situación de evaluar a sus colaboradores más cercanos. El resultado es un sistema de evaluación generalmente distorsionado, con evaluaciones infladas y sutiles sistemas de rotación para la asignación de calificaciones (Shafritz et al., 1992). También se puede desvirtuar el sistema en el sentido contrario, ya que hemos visto que en circunstancias de baja afinidad con sus subordinados, los políticos tienen incentivos a bajar el nivel de las evaluaciones.

\subsection{El afecto y las preferencias personales}

Las cuestiones afectivas y las preferencias personales afectan notablemente los resultados de las evaluaciones. De acuerdo a la evidencia empírica en este sentido, la preferencia personal por el subordinado tiende a incrementar el efecto halo, si bien el desagrado no parece tener un efecto tan directo sobre las evaluaciones. Villanova y Bernardin (1989) proponen un modelo para explicar el manejo de impresiones en la evaluación de desempeño en el cual el nivel de agrado ("liking") actúa como variable mediadora entre el comportamiento oportunista y el resultado de la evaluación. Las condiciones que favorecen la simpatía, actúan indirectamente en forma positiva sobre el resultado de la evaluación.

El grado de similitud interpersonal entre evaluador y evaluado afecta el nivel de agrado, y por 10 tanto, los resultados de la evaluación de desempeño. A mayor grado de similaridad de actitudes y valores, es mayor la simpatía, y mayor la calificación (Ferris et al., 1994). Partiendo de esta afirmación, se podría plantear como 
hipótesis una tendencia a evaluaciones deflacionadas en el caso de políticos evaluando a funcionarios técnicos, basado en la diferenciación de ambos grupos. Aderbach y sus colegas (1981) plantean las diferencias entre los dos grupos en términos de origen social, educación, patrones de reclutamiento y antigüedad en el servicio. Los políticos son designados en base a la confianza política, y en segundo término por su experiencia previa en el área de que se trate. El caso de los funcionarios de línea es el opuesto, ya que son seleccionados en base a principios de mérito, según sus antecedentes y sus habilidades específicas. Dada la importancia de los antecedentes de formación en su designación, los funcionarios de línea tienen más altos niveles de educación formal que los políticos.

Estas diferencias se reflejan en las opiniones mutuas entre funcionarios de línea y funcionarios políticos, que no suelen ser demasiado positivas en lo que hace a efectividad para el desempeño de la función. En este sentido es interesante ver los resultados del informe sobre el Senior Executive Service (SES) realizado por el Merit Systems Protection Board (1987). Los resultados de la encuesta administrada a ex-funcionarios del SES mostraron que los funcionarios de carrera no tienen un gran concepto respecto de las habilidades gerenciales de los políticos, si bien reconocen su rol en la formulación de políticas. Esta opinión contrasta con la evaluación marcadamente positiva que los políticos hicieron de sus propias habilidades como gerentes. Otro resultado interesante es que los funcionarios de carrera supervisados por políticos tienen una opinión menos positiva de su supe- rior que los supervisados por funcionarios de carrera. Los funcionarios cuyo último supervisor fue un político expresaron su preocupación acerca de la competencia de su superior y de la politización de sus organizaciones.

La distancia psicológica entre evaluador y evaluado es importante también. A mayor distancia, menor nivel en la calificación del subordinado. Una medida utilizada para valorar la distancia en este sentido es el tiempo durante el cual evaluador $y$ evaluado han interactuado previo al momento de la evaluación. A menos cantidad de tiempo, mayor la distancia psicológica, y mayor la probabilidad de evaluaciones deflacionadas. En el caso de funcionarios de línea y funcionarios políticos este es un punto crítico. Los funcionarios de línea son reclutados para los puestos jerárquicos mediante una carrera progresiva interna a la organización, mientras que los políticos tienen un alto grado de ingreso lateral desde otras instituciones. La rápida rotación de los cargos políticos hace que se dificulte la consolidación de un sentido de equipo de trabajo, y de una confianza sólida entre los dos grupos, lo cual podría generar una tendencia a evaluaciones devaluadas en los casos en que no hay un conocimiento previo del evaluado.

\subsection{Imagen del proceso de evaluación}

Un factor importante que afecta el comportamiento político de los evaluadores es el grado de confianza en el proceso de evaluación. Si hay una creencia generalizada acerca de la seriedad del proceso, y los supervisores tienen que responder por los resultados de las evaluacio- 
nes, habrá un menor nivel de actividades de influencia. Consideraciones de equidad también afectan la actitud de los evaluados en términos del nivel de influencia hacia el proceso de evaluación. Cuando el sistema se considera injusto, habrá mas intentos de influir las evaluaciones por medios políticos (Milgrom \& Roberts, 1992).

\section{Cómo controlar la politización de la evaluación de gerentes en el Estado?}

Como hemos visto, el proceso de evaluación de desempeño en sí mismo es objeto de influencia política debido a la importancia de las decisiones que involucra. Es un proceso complejo, orientado a objetivos múltiples, en el cual los participantes tienen intereses personales involucrados. Este es el marco ideal para el desarrollo de actividades de influencia.

De acuerdo a los argumentos expuestos en las secciones anteriores, el nivel de actividad política en un determinado proceso de evaluación de desempeño dependerá de distintas variables. Habrá más actividad política cuando:

- consecuencias importantes para las partes dependen de la evaluación,

- las evaluaciones se realizan con poca frecuencia,

- se genera incertidumbre por basarse la evaluación en criterios subjetivos

- se trata de un proceso poco formalizado

- los medios políticos son útiles para lograr los objetivos personales

- se trata de los niveles jerárquicos superiores de la organización
- el grado de afinidad entre evaluadory evaluado es bajo

- el grado de confianza y equidad percibida del proceso de evaluación es bajo.

El grado de politización del proceso podría afectarse entonces mediante la manipulación de cada uno de estos factores. Veamos entonces cada uno de los factores analizados.

\subsection{Limitar la importancia del proceso de evaluación}

Una posibilidad para limitar el nivel de politización es trabajar sobre el nivel de importancia del proceso de evaluación. Tal como indican Milgrom \& Roberts (1992), se podrían limitar los efectos redistributivos de la decisión a tomar para reducir los incentivos al desarrollo de actividades de influencia. En el contexto del proceso de evaluación de desempeño, esto significaría no basar el pago, las promociones y otras decisiones importantes en los resultados de la evaluación.

Pero el caso es analizar hasta que punto es posible $-y$ eficiente- para una organización implementar una política que le impediría utilizar información valiosa para tomar decisiones centrales; más aún en las organizaciones públicas, cuyo sistema de recursos humanos se debe basar justamente en los principios de mérito. Las decisiones se basan en el desempeño o mérito de los individuos precisamente por las dificultades que presentaría definir medidas de resultado en la mayoría de las organizaciones públicas, con lo cual se hace difícil limitar la relación entre evaluación de desempeño y sistema de recompensas. Si se desdibuja la relación entre la evaluación obtenida y 
sus consecuencias se reducen los incentivos a la actividad política, pero también se desvirtúa el mecanismo básico de motivación en los sistemas de mérito.

En las organizaciones públicas entonces, el sistema de evaluación de desempeño es central para la distribución de recursos. Abrir el proceso de decisión a la participación de individuos que tienen sus propios intereses en los resultados aumenta los costos de influencia, especialmente cuando la decisión redistribuye recursos valiosos entre los participantes. Al mismo tiempo, estos costos tienen que ser balanceados con la mejora en la información que acompaña esta participación de los interesados. Este es claramente el caso en los procesos de evaluación de desempeño. La información provista por el supervisor y por el evaluado es muy valiosa para la organización para tomar decisiones centrales, pero el proceso de obtención de esa información estará sujeto a actividades de influencia por ambas partes. Una alternativa para evitar la politización sería también prescindir totalmente de la evaluación del supervisor político, y basar las decisiones sobre la carrera del funcionario en una evaluación totalmente externa. Si bien la solución parece atrayente, la mejor observación del desempeño se obtendrá desde dentro de la organización, y la visión del supervisor directo es fundamental para lograr una descripción adecuada del mismo.
Dado que no parece razonable dejar de utilizar la información obtenida en las evaluaciones de desempeño para decisiones tan importantes, una alternativa para reducir el nivel de importancia otorgada al proceso por parte del evaluador $y$ del evaluado es aumentar la frecuencia de las evaluaciones. El problema es que tiene que haber un ajuste entre el momento en que se evalúa y el momento en que se utiliza la información para promociones y remuneraciones, y esas decisiones tienen generalmente un ciclo anual. Además, una evaluación formal significa una inversión de tiempo importante, y aumentar su frecuencia significaría distraer a evaluadores y evaluados de sus tareas específicas.

Una propuesta interesante sería implementar un sistema de feedback frecuente que complemente la evaluación anual. El nivel de feedback afecta las actitudes de los empleados respecto del trabajo, y sus percepciones respecto de los sistemas de evaluación y recompensas. Yeager, Rabin y Vocino (1985) estudiaron la relación entre estas variables en posiciones administrativas en el sector público en Estados Unidos ${ }^{7}$ y encontraron que menores niveles de feedback llevaban a menores niveles de comprensión del sistema de evaluación, menor grado de aceptación de los criterios de evaluación y satisfacción con los resultados, y menor percepción de justicia del sistema. Por otro lado, Birkenhoff y Kanter (1980)

7 El estudio se basa en posiciones administrativas, incluyendo los niveles federal, estatal y local. La base de datos utilizada es el National Longitudinal Study (NLS) of Political Science, Public Administration and Public Affairs graduates. 
sostienen que la falta de un sistema de feedback formal también provee a los evaluadores de una posibilidad de manipular las evaluaciones, ya que pueden negar a sus subordinados el feedback que necesitarían para mejorar su desempeño y lograr mejores calificaciones.

Si no hay un adecuado sistema de feedback, se genera un clima de escepticismo acerca del sistema de evaluación y de la manera en que se obtienen las recompensas, reforzando los incentivos para las actividades de influencia sobre el proceso de evaluación. Un sistema de feedback frecuente en el contexto de la evaluación de funcionarios de línea por parte de políticos reforzaría los vínculos entre ambos grupos, y brindaria un espacio donde dirimir las divergencias en cuanto a los objetivos y las tareas a realizar.

\subsection{Reducir la incertidumbre}

Otra alternativa sería reducir la incertidumbre del proceso, mediante la utilización de criterios objetivos de evaluación, y una definición clara de los objetivos esperados. Ya se ha hecho referencia a las dificultades que plantean estos temas en el caso de altos niveles en las organizaciones, especialmente en el sector público. Sin embargo, la experiencia del Federal Civil Service en cuanto al desarrollo de estándares de evaluación a partir de la Civil Service Reform Act (Ley de Reforma del Servicio Civil) de 1978 muestra una estrategia exitosa en este sentido (GAO, 1983). La ley de reforma ordenaba a las agencias federales desarrollar criterios objetivos para la evaluación de sus empleados, insistiendo en que el sistema de evaluación debía incluir la explicitación acerca de los estándares a partir de los cuales se tomarian las decisiones en el proceso de evaluación. El establecimiento de estándares de evaluación debía realizarse en forma consensuada entre evaluadores y evaluados. $\mathrm{Si}$ bien el proceso presentó muchas dificultades, se logró en cierta forma la objetivación de los criterios de evaluación. Un beneficio adicional de este proceso fue la mejora en la comunicación interpersonal, que disminuyó las tensiones relacionadas con las evaluaciones.

\subsection{Formalizar el proceso de evaluación}

Otro camino posible para disminuir las actividades de influencia es reducir la autonomía del superior en la toma de decisiones. En principio, la formalización del mismo, a partir del establecimiento de reglas y procedimientos precisos, disminuye la incertidumbre respecto del mismo $y$ deja poco espacio para posibles arbitrariedades por parte del supervisor. Se han desarrollado instrumentos de evaluación específicamente orientados a evitar manipulaciones por parte de los evaluadores, tales como el método de "elección forzada", o las "escalas de estándares mezclados"8. El problema con estos mecanismos es que al mismo tiempo que evicada ítem, para que el evaluador no sepa cual de las alternativas favorece más al subordinado. 
tan arbitrariedades, reducen la efectividad del sistema de evaluación como herramienta de gerenciamiento y pueden generar un sistema injusto. Ninguno de estos mecanismos permite al evaluado conocer con certeza los criterios a partir de los cuales será evaluado. En Estados Unidos, desde la reforma del servicio civil en 1978 (CSRA) no se pueden utilizar estos métodos, porque la ley exige que se comunique a los empleados los estándares de evaluación al principio del período a evaluar.

Otra forma de reducir la autonomía del político en la evaluación del funcionario de línea es hacerlo responsable ante niveles superiores de los resultados de las evaluaciones, estableciendo una revisión de las mismas. Para que resulte efectiva debe aclararse bien el rol del revisor, ya que de lo contrario se tranforma en una mera formalidad para confirmar las decisiones ya tomadas ${ }^{9}$. En el caso de evaluaciones en los máximos niveles del Estado se puede asignar el rol de revisión a un equipo técnico interno o externo, o también a un grupo de pares. El Senior Executive Service en los Estados Unidos utiliza la evaluación por pares para complementar la evaluación por parte del superior político para evitar decisiones arbitrarias. Sin embargo los re- caudos no parecen ser suficientes, ya que los miembros del SES reportan un nivel significativo de interferencia política en las decisiones administrativas de los organismos donde se desempeñan (MSPB, 1987).

\subsection{Reforzar la credibilidad del sistema de evaluación}

También se puede reducir la politización del sistema de evaluación tomando medidas que refuercen la credibilidad del sistema y la percepción de justicia acerca del mismo por parte de evaluadores y evaluados. La evaluación de desempeño no tiene que ser vista como una instancia burocrática de llenado de formularios, ni como un espacio de confrontación entre subordinado y superior. Estas situaciones llevan a que se desvirtúen los objetivos de la evaluación de desempeño.

El tema de la confianza en el sistema y en la percepción de justicia acerca de las evaluaciones es recurrente en todos los estudios sobre el Federal Civil Service en Estados Unidos. Estos estudios coinciden en que el nivel de confianza de los individuos respecto de la organización determina el grado de aceptación del sistema. El sistema de evaluación de desempeño tiene que ser aceptado por

Este método se utilizó durante un tiempo en la armada de los Estados Unidos. En las "escalas desordenadas de estándares" (mixed standard scales) se provee al evaluador con una lista de afirmaciones que reflejan tres niveles de cada comportamiento evaluado. Estas afirmaciones están desordenadas de modo que el orden de mérito dentro de cada tríada no es obvio.

9 Este es uno de los problemas identificados por la General Accounting Office respecto de la implementación del sistema de evaluación de desempeño establecido en la reforma del servicio civil. (GAO, 1983) 
las partes, y ser confiable respecto de los resultados que produce (GAO, 1993). Las recomendaciones para aumentar el nivel de confianza en el sistema se basan casi siempre en la participación activa de evaluador y evaluado en la definición de las reglas del juego.

La participación del evaluado en el establecimiento de los estándares respecto de los cuales será evaluado genera un mayor compromiso con el sistema de evaluación, y en principio se podría esperar que disminuyan los incentivos para comportamientos políticos de su parte. La administración por objetivos parecería plantear una solución para impulsar la credibilidad en los sistemas de evaluación. Sin embargo, el hecho de negociar los objetivos de la gestión también puede ser objeto de estrategias de influencia. De esta manera, el juego político se trasladaría al establecimiento de los objetivos (Fink D., 1994). Los gerentes podrían establecer objetivos fácilmente alcanzables para lograr obtener sus recompensas, 0 podrían fijar objetivos demasiado altos como una estrategia para justificarse si no se cumple con lo planeado.

Además de la manipulación del nivel a alcanzar respecto de los objetivos, también es posible que los objetivos determinados no tengan relación directa con la misión de la organización, con lo cual se desvirtúa el sistema. Osborne y Gaebler (1992) proponen la administración por objetivos como mecanismo interno de control de las organizaciones públicas. En principio la administración por objetivos debería lograr que todos los procesos internos en las organizaciones públicas estén orientados a la misión de la organización, y en último término a los clientes. Steinberg y Burke (1986) plantean que si el proceso se desarrolla en forma de "cascadas de objetivos" se evitan los problemas de distorsión mencionados. El máximo nivel en la organización debería determinar los objetivos globales asociados con la misión, y comunicarlo hacia los niveles inferiores que deberían operacionalizarlos cada vez más a medida que se desciende hacia los niveles operativos. Aplicado de esta manera, sin perder la conexión con la misión de la organización, el sistema de administración por objetivos puede resultar un instrumento valioso para reducir la actividad política en la evaluación de desempeño.

\subsection{Crear incentivos que desalienten las actividades de influencia}

También es importante trabajar sobre el desarrollo de una cultura en la cual no se premie la utilización de actividades de influencia. El tema es como se define el concepto de responsabilidad (accountability) en la organización, y como se operacionaliza con respecto a las evaluaciones. Jorgensen y sus colegas (1996) hacen un interesante análisis del cambio planteado a partir del National Performance Review en el Federal Civil Service de los Estados Unidos. Este programa plantea un cambio del tradicional sistema de responsabilidad basado en los principios de mérito, instalando un sistema de responsabilidad en términos de resultados hacia el público o hacia sus clientes internos en el Estado. Se enfatiza la descentralización, desregulación, simplificación y flexibilidad, en el marco de un incremento sustancial del nivel de delega- 
ción de autoridad. Si bien se indica que la responsabilidad hacia el cliente debe lograrse "adhiriendo a principios de mérito, equidad e igualdad de oportunidades", muchas veces los superiores se encontrarán ante la disyuntiva entre los dos principios. En el actual contexto del Federal Civil Service, los gerentes a cargo de procesos de evaluación de desempeño buscarán la manera de lograr que los resultados de los mismos sean funcionales a sus necesidades en términos de los resultados hacia afuera, mas que cuidar el cumplimiento estricto de las normas formales que regulan el proceso.

\section{Conclusiones}

La perspectiva política planteada permite una descripción acertada del proceso de evaluación de desempeño en altos niveles en el gobierno. A partir de sus supuestos, parecerían inútiles todos los esfuerzos por mejorar la precisión de los métodos de evaluación de desempeño, ya que el proceso en sí está orientado a objetivos que tienen poco que ver con la descripción objetiva del desempeño. Si bien el modelo tiene un objetivo descriptivo y no prescriptivo, a partir del análisis de los antecedentes de las actividades de influencia se pueden inferir estrategias para acercar el sistema de evaluación de desempeño al ideal del modelo racional.

En este trabajo se exploraron distintas alternativas para reducir el nivel de politización de la evaluación de gerentes en el Estado, trabajando sobre los antecedentes de las actividades de influencia. Algunas de estas alternativas entran en conflicto con los objetivos de la evaluación de desempeño. Por ejemplo, limitar totalmente la discreción del evaluador le impide utilizar la evaluación como herramienta de gerenciamiento. La solución extrema de descartar todo tipo de evaluación formal de los niveles superiores en el Estado tampoco parece ser la respuesta al problema, ya que el sistema de recursos humanos está definido como un sistema de mérito, y la evaluación de desempeño es el mecanismo que permite identificar la contribución de cada individuo o grupo de trabajo a la organización.

En definitiva, hay que crear los incentivos suficientes para que los beneficios de producir evaluaciones precisas sean más altos que sus costos tanto para el evaluador como para el evaluado. La alternativa de implementar sistemas de feedback permanente en forma paralela a las evaluaciones anuales puede resultar un método efectivo para reducir la politización del proceso. La implementación de sistemas de evaluación en el marco de la administración por objetivos resultaría en una objetivación de los estándares de desempeño, y permitiría un mayor compromiso de los políticos y funcionarios de línea con el sistema de evaluación. Mecanismos de revisión de las evaluaciones de los superiores políticos por parte de equipos técnicos, o la inclusión de la evaluación de pares para complementarlas pueden ser alternativas interesantes para limitar la discrecionalidad de los evaluadores, sin anular su contribución al proceso de evaluación.

Si bien el modelo político provee una buena descripción de la realidad, el modelo racional y su objetivo de precisión en la medición del desempeño provee el ideal teórico del proceso de evaluación. La pregunta relevante involucra a ambos 
modelos, y consiste en platearse como maximizar la precisión del sistema de evaluación dados los procesos políticos subyacentes. Para esto es necesario centrarse en las características de la organización y del proceso en sí, que favorecen el desarrollo de actividades de influencia, para identificar las variables a manipular a fin de lograr un proceso de evaluación más objetivo.

\section{Bibliografía citada}

Aderbach, J.D., Putnam, R.D. \& Rockman B.A. (1981) Bureaucrats and politicians in Western democracies. Cambridge, MA: Harvard University Press.

Birkenhoff, D.W. \& Kanter, R.M. (1980) Appraising the performance of performance appraisal. Sloan Management Review, Spring 1980, 3-16.

Cardy, R. \& Dobbins, G. (1994) Performance Appraisal: Alternative Perspectives. South Western Series in Human Resource Management.

Daley, D.M. (1992) Performance appraisal in the public sector. Techniques and applications. Wesport, CT: Quorum Books.

Dwight, D.F. (1994) Accountability in Human Resource systems: the impression management and performance directed functions of goal settting in the performance evaluation process. PhD Thesis. University of Illinois at Urbana-Champaign.

Ferris, G.R. \& Judge, T.A. (1991). Personnel/ Human Resources Management: A Political Influence Perspective. Journal of Management, 17. 447-488.

Ferris, G.R., Judge, T.A., Rowland, K. \& Fitzgibbons, D. (1994) Subordinate Influence and the Performance Evaluation Process: Test of a Model. Or- ganizational Behavior and Human Decision Processes. 58. 101-135.

Ferris, G.R., Russ, G.S., \& Fandt, P.M. (1989). Politics in organizations. In R.A. Giacalone and P. Rosenfeld (Eds.), Impression management in the organization ( $p p$ 143-170). Hillsdale, NJ: Lawrence Erlbaum.

General Accounting Office (1983) New Performance Appraisals Beneficial but Refinements Needed. Washington, DC.

General Accounting Office (1993) Federal Performance Management: Agencies need greater flexibility in designing their systems. Washington, DC, February 1993.

Gore, A. (1993) From Red Tape to Results. Creating a Government that works better and costs less. Reinventing Human Resource Management. Washington: DC, September 1993.

lacoviello, M. (1996) El juego político y las estrategias de recursos humanos en el Estado. Cuadernos CEPAS, No 4.

Jorgensen, L.; Fairless K. \& Patton, W.D. (1996) Underground merit systems: balance between service and compliance. Review of Public Personnel Administration Spring 1996, vol. xvi, no.2

Longenecker, C.O. (1989). Truth or consequences: Politics and performance appraisais. Business Horizons, 32, 76-82.

Longenecker, C.O.; Gioia, D.A. \& Sims, H.P. (1987). Behind the Mask: The Politics of Employee Appraisal. Academy of Management Executive, Vol 1, No. 3., 183-193.

Merit System Protection Board (1987) The Senior Executive Service, views from former federal executives. A Report to the President and the Congress of the United States by the US MSPB. 
Milgrom, P. \& Roberts, J. (1992) Economics, Organizations \& Management. Englewood Cliffs, NJ: Prentice-Hall.

Mintzberg H. (1983) Power in and around organizations. Englewood Cliffs, $\mathrm{NJ}$ : Prentice-Hall.

Murphy, K.R. \& Cleveland, J.N. (1995) Understanding Performance Apprajsal: Social, Organizational and Goal-Based perspective. Thousand Oaks, CA: SAGE Publications.

Osborne, D.; Gaebler, T. (1992) Reinventing government: How the entrepreneurial spirit is transforming the public sector. Reading, MA: Addison-Wesley.

Pfeffer, J. (1992) Managing with power. Boston, MA: Hanvard Business School Press.

Salancik G.R. \& Pfeffer J. (1976) Who gets the power and how they hold it: a strategic contingency model of power. Organizational Dynamics. Winter 1977. American Management Association, NY.

Shafritz, J.M., Ricucci, N.M., Rosenbloom, D.H. \& Hyde, A.C. (1992) Personnel management in government: Poli- tics and process. (fourth edition revised). New York: Marcel Dekker, Inc.

Steinberg, A. \& Burke, W. (1986) Recommendations for improved performance appraisal in the federal sector. US Army Research Institute for the Behavioral and Social Sciences.

Villanova, P.D. \& Bernardin, H.J. (1989). Impression Management in the Context of Performance Appraisal. In R.A. Giacalone, \& P. Rosenfeld (Eds.) Impression Management in the Organization. (pp.299-313). Hilldsdale, N.J.: Lawrence Erlbaum Associates, Publishers.

Welsh, M.A. \& Slusher E.A. (1986) Organizational Design as a context for political activity. Administrative Science Quarterly, 31, 389-402.

Wood, R., \& Mitchell, T. (1981). Manager behavior in a social context: The impact of impression management on attributions and disciplinary actions. Organizational Behavior and Human Performance, 28, 356-378.

Yeager, S.; Rabin, J.; \& T. Vocino (1985) Feedback and administrative behavior in the public sector. Public Administration Review, Vol. 45, 570-75. 